\title{
Honey as complementary and alternative medicament in pulpotomy of permanent teeth
}

\begin{abstract}
Background/objectives: Honey is being used as complementary and alternative medicine since ages. So far, scientific evidence has also proven its beneficence in different human diseases. Its use in dentistry as antimicrobial, analgesic and antiinflammatory has also been well established. Although many clinically effective dressing materials for pulpotomy are available in the market, but scientists are still in search of an ideal material having more beneficial effects, less harms and moreover, cost effective. Therefore, a study was planned to describe and compare inflammatory response of human dental pulp tissue on application of dressings of calcium hydroxide, honey and a mixture of calcium hydroxide and honey.
\end{abstract}

Material and methods: For this experimental study, 36 human premolar teeth scheduled to be extracted for orthodontic reasons, from 12 patients were selected. After informed consent, pulpotomy procedure was carried out and dressings were placed. The inflammatory grades were noted under light microscope after histological processing of extracted teeth

Results: After 7 days, in Ca-hydroxide group, $5(83.3 \%)$ in grade-II and $1(16.7 \%)$ in grade-I and in honey group 3(50.0\%) in grade-II and 3(50.5\%) also in grade-I but in Calcium hydroxide + Honey only $1(16.7 \%)$ is in grade-II and $5(83.7 \%)$ are in grade-I which indicates that honey with Ca-hydroxide has more anti-inflammatory activity as compared to Ca-hydroxide or honey alone after pulpotomy

Conclusion: Honey alone and Ca-hydroxide alone almost have similar antiinflammatory potential, but when they are used in combination, they act as more potent anti-inflammatory agents after pulptomy.

Keywords: raw honey dressing, $\mathrm{CaOH}$, root canal treatment, pulpotomy, pathology
Volume 3 Issue 2 - 2016

\author{
Ayesha Bshir,' Shazia Naz,' Kashif Adnan, ${ }^{2}$ \\ Babra Marium Anwari² \\ 'Department of Operative Dentistry, de'Montmorency College \\ of Dentistry Lahore, Pakistan \\ ${ }^{2}$ Department of Operative Dentistry, de'Montmorency College \\ of Dentistry Lahore, Pakistan
}

\section{Correspondence: Ayesha Bashir, Department of Operative Dentistry, de'Montmorency College of Dentistry, 54000, Lahore, Pakistan, Tel 00923014366 278,}

Email themedresearcher@gmail.com

Received: October 30, 2016 | Published: November 18, 2016

\section{Introduction}

For the maintenance of integrity of dental arches, the preservation of tooth vitality is very important. If a tooth is damaged by bacterial attack or certain other reason, a dentist has to make decisions about what procedure should be adopted and this depends upon the extent of pulpal damage. ${ }^{1}$ If damage is of less extent, i.e. when coronal pulp is damaged due to caries, traumatic injury to the tooth and other iatrogenic causes, the preferred method in such cases is pulpotomy. One very critical thing that should be kept in mind that radicular pulp should be free of any pathology.

For decades, a plethora of medicaments are used by the dentists in pulpotomy procedures, both in deciduous and permanent teeth. One of the cost effective and clinically successful agent is calcium hydroxide (Ca-hydroxide) in context of pulpotomy. ${ }^{2}$ During the past several decades, form cresol is a famous compound as pulpotomy dressing, especially for the primary molars. ${ }^{3}$ Now a days, its use become limited as pulpotomy medicament because of reported mutagenic and carcinogenic effects. ${ }^{4-6}$

Due its antimicrobial, antioxidant, sterility and inflammation, relieving characteristics, honey are being used since ages. Even medical professional of today admires the its use for infected wounds, treatment of burns and ulcers. ${ }^{7,8}$ In dentistry, it is documented in the literature that it has useful effects in gingivitis, periodontitis, halitosis etc. ${ }^{9}$ Honey reduces the incidence of mucositis in patients receiving radiation therapy for oral cancer as well as it has antibacterial and analgesic properties when used a root canal medicament. ${ }^{10,11}$ Honey in combination with mustard oil has been advocated as an available alternate endodontic medicament. ${ }^{12}$ It has been reported in another study that natural honey showed antibacterial action against anaerobic bactericides present in dental abscess and osteomyelitis. ${ }^{13}$ Honey clear infections, removes malodour, reduces inflammation and pain, causes oedema and exudation to subside, and increases the rate of healing by stimulation of angiogenesis, granulation and epithelization. ${ }^{7,8}$ As the honey is available all across the world at a very nominal cost, research should be carried out on its potential therapeutic role in endodontic dental treatments. It is hypothesized that dressing of a mixture of calcium hydroxide with honey may produce better inflammatory response for the healing of human dental pulp. Take into account the documented literature, a study was designed to evaluate the response of dental pulp tissue after application of calcium hydroxide, honey and a mixture of calcium hydroxide and honey as dressing material.

\section{Material and methods}

\section{Sample selection}

This Quasi experimental study was carried out in the Operative Dentistry Department, de'Montmorency College of Dentistry, Lahore, Pakistan in January-June 2015. Thirty six_human first premolar teeth from twelve patients of age ranging between 15-25years. Teeth were divided into 3 groups of 12 teeth in each group. 
Patients were selected by following convenient, non- probability sampling technique. Three first premolar teeth in every patient were filled after pulpotomy, with materials of each group, so that these materials could be evaluated in same oral environment.

i. Group I: Pulpotomy with $\mathrm{Ca}(\mathrm{OH})^{2}$ dressing

ii. Group II: Pulpotomy with honey dressing

iii. Group III: Pulpotomy with honey $+\mathrm{Ca}(\mathrm{OH})^{2}$ dressing (in equal proportion)

\section{Inclusion criteria}

i. Premolar teeth scheduled to be extracted for orthodontic reasons were selected from patients ranging from 15-25years old with good general and oral health.

ii. All teeth were examined clinically and radiographically to assure the absence of proximal caries and periapical lesions.

iii. All vital teeth were assessed by the electrical pulp testing method.

\section{Exclusion criteria}

i. Teeth with abnormal sensitivity to heat and cold, chronic pulpagia, tenderness on percussion or palpation because of pulpal disease.

ii. Teeth with periradicular radiographic changes resulting from extension of pulpal diseases into periapical tissues.

iii. Teeth with second and third grade mobility.

\section{Data collection procedure}

After fulfilling the inclusion and exclusion criteria, local anesthetic was injected. Each tooth was cleaned with a nylon brush and a polishing paste and then washed with $70 \%$ alcohol solution. After rubber dam application cavities were prepared on occlusal surfaces of the thirty six premolar teeth using sterile diamond burs \#310, mounted on a high speed turbine with sufficient cooling water. The cavities were rectangular, $4 \mathrm{~mm}$ long mesiodistally, $2.5 \mathrm{~mm}$ wide buccolingually and $3.5 \mathrm{~mm}$ deep to remove the roof of the pulp chamber. Hemorrhage was controlled with a moist pledget of cotton. The cotton pledgets were renewed till the complete hemostasis was established. Coronal pulp was removed with a long shank spoon excavator and irrigated the cavity with anesthetic solution and then dried it with a sterile dry cotton pledget. After hemostasis, dressings were placed on amputated pulps. For Group-I, Calcium hydroxide powder was mixed in sterile water to a thick consistency on a glass slab with the aid of cement spatula. The mixture was carried to the amputated pulp using a plastic instrument. In group II, Dressing of raw honey was applied on amputated pulp with the help of plastic instrument. In group III, calcium hydroxide was mixed in honey (in equal proportion) with a cement spatula on a glass slab to a thick consistency. The mixture was carried to the amputated pulp with the help of plastic instrument.

After the placement of these dressing materials, teeth were restored with composite resin using glass ionomer cement as a liner. Teeth of six patients (age 15-25years) were extracted after one week and the other six after four weeks for the subsequent procedure. Hence six teeth in each group extracted after one week and the other six teeth after four weeks. Tooth roots were prepared in sections of about $2 \mathrm{~mm}$ to facilitate fixation in $10 \%$ buffered formal for seventy two hours.
Teeth were decalcified in $20 \%$ formic acid for six to eight weeks, and were prepared in accordance with normal histological techniques and embedded in paraffin. Six micron sections were cut with microtome parallel to the main vertical axis of the tooth. The sections were mounted on glass slides and were stained with haematoxylin and eosin. The stained sections were seen microscopically to observe any kind of pulpal cell response. The sections were evaluated under supervision of experienced pathologists and calibrated according to the criteria described as follows:

\section{Inflammatory cell score}

i. Grade I: Absent or very few inflammatory cells.

ii. Grade II: Mild or average number less than ten inflammatory cells.

iii. Grade III: Severe inflammatory lesion appearing as an abscess or dense infiltrate involving one third or more of the pulp.

iv. Grade IV: Completely necrotic pulp.

\section{Statistical analysis}

The clinical and histological data were entered and analyzed using the SPSS 20.0. Fisher Exact Test was applied to see the statical association in all the three study groups. A $p$ value of $\leq 0.05$ was considered as statistically significant. This study was approved by the Ethical Review Committee and Advanced Studies \& Research Board, University of Health Sciences, Lahore, Pakistan.

\section{Results}

The results after one week of pulpotomy, show that there is no statistically significant association $(\mathrm{p}>0.05)$ between groups and grades of inflammation. It demonstrates that Ca-hydroxide, Honey and Calcium hydroxide+Honey groups, no significant differences in the grades of inflammation were found on descriptive statistics. After 7 days 6 teeth were extracted in each group $(\mathrm{CaOH}$, Honey and $\mathrm{CaOH}+\mathrm{Honey})$, in Ca-hydroxide group, $5(83.3 \%)$ in grade-II and 1 out of $6(16.7 \%)$ in grade-I and in honey group 3 out $6(50.0 \%)$ in grade-II and $3(50.5 \%)$ also in grade-I but in Calcium hydroxide+Honey only $1(16.7 \%)$ is in grade-II and $5(83.7 \%)$ are in grade-I which indicates that honey with Ca-hydroxide has more anti-inflammatory activity as compared to Ca-hydroxide or honey alone after pulpotomy (Table 1).

Similar findings have been observed after 4 weeks, we can see here that Fisher's Exact Test shows insignificant statistical association after 4 weeks again $(\mathrm{p}=0.589)$. This finding shows that there is no statistically significant association among groups and grades of inflammation. That is, in Ca-hydroxide, Honey and Ca-hydroxide+Honey groups the proportion of inflammatory grades does not vary widely. After 28 days remaining 6 teeth in each group were extracted, in Ca-hydroxide group, $4(66.7 \%)$ in grade-II and $2(33.3 \%)$ in grade-I and in honey group $4(66.7 \%)$ in grade-II and $2(33.3 \%)$ also in grade-I, but in Cahydroxide+Honey group only $2(33.3 \%)$ are in grade-II and $4(66.7 \%)$ are in grade-I which indicate that honey with Calcium hydroxide has more effect in reduction of inflammation (Table 1).

We can now summarize the findings that honey alone and $\mathrm{Ca}$ hydroxide alone almost have similar anti-inflammatory potential, but when they are used in combination, they act as more potent antiinflammatory agents after pulptomy (Table 2). 
Table I Descriptive statistics after one week and four weeks: Grading of inflammation among groups

\begin{tabular}{lllllll}
\hline \multirow{2}{*}{ Group } & & \multicolumn{2}{l}{ Score } & \multicolumn{2}{c}{ Total } \\
\cline { 3 - 6 } & Grade I & Grade 2 & Grade 3 & Grade 4 & \\
\hline \multirow{2}{*}{7 days } & Ca-hydroxide alone & I (17\%) & $5(83 \%)$ & 0 & 0 & 6 \\
& Honey alone & $3(50 \%)$ & $3(50 \%)$ & 0 & 0 & 6 \\
& Ca-hydroxide+Honey & $5(83 \%)$ & $1(17 \%)$ & 0 & 0 & 6 \\
28 days & Total & 9 & 9 & 0 & 0 & 18 \\
& Ca-hydroxide alone & $2(33 \%)$ & $4(67 \%)$ & 0 & 0 & 6 \\
& Honey alone & $2(33 \%)$ & $4(67 \%)$ & 0 & 0 & 6 \\
& Ca-hydroxide+Honey & $4(67 \%)$ & $2(33 \%)$ & 0 & 0 & 6 \\
& Total & 8 & 10 & 0 & 0 & 18 \\
\hline
\end{tabular}

Table 2 Summary of descriptive statistics: Inflammatory grades among groups

\begin{tabular}{|c|c|c|c|c|c|c|}
\hline Group & & Grade-I & Grade-II & Grade-III & Grade-IV & Total \\
\hline \multirow[t]{2}{*}{1} & Count & 3 & 9 & 0 & 0 & 12 \\
\hline & $\%$ within group & $25.00 \%$ & $75.00 \%$ & $0 \%$ & $0 \%$ & $100.00 \%$ \\
\hline \multirow[t]{3}{*}{2} & Count & 5 & & 0 & 0 & 12 \\
\hline & $\%$ within group & $41.70 \%$ & 7 & $0 \%$ & $0 \%$ & $100.00 \%$ \\
\hline & & & $58.30 \%$ & & & \\
\hline \multirow[t]{3}{*}{3} & Count & 9 & & 0 & 0 & 12 \\
\hline & $\%$ within group & $75.00 \%$ & 3 & $0 \%$ & $0 \%$ & $100.00 \%$ \\
\hline & & & $25.00 \%$ & & & \\
\hline \multirow[t]{3}{*}{ Total } & Count & 9 & & 0 & 0 & 12 \\
\hline & $\%$ within group & $47.20 \%$ & 3 & $0 \%$ & $0 \%$ & $100.00 \%$ \\
\hline & & & $58.20 \%$ & & & \\
\hline
\end{tabular}

\section{Discussion}

Now it has been proved that remnants of vital pulp tissue can regenerate the whole dental pulp, therefore, maintaining the tooth vitality is very important. Pulpotomy comprised of cutting inflamed or infected coronary pulp and capping the remaining vital pulp tissue with a material that maintains its vitality. This vital pulpal therapy has been strongly recommended for young permanent teeth, especially in clinical situations when a large pulp exposure is caused by decay or trauma or when the root is not completely formed. ${ }^{14}$

In the current study, a comparison was made among antiinflammatory potential of $\mathrm{CaOH}$ alone, Honey alone and honey $+\mathrm{CaOH}$ combination as dressing materials after pulpotomy. The clinicians generally agree that pulp capping is clinically successful if the tooth functions free of symptoms reacts adequately to sensitivity tests and has a normal radiographic appearance. It is impossible to clinically diagnose teeth in which healing is complicated by inflammation. Therefore, a critical evaluation of the results of vital pulp therapy can only be made for this reason, more sensitive methods and/or instruments need to be developed. ${ }^{15}$

In the present study, specimens (premolars) given the dressings of honey exhibited less inflammation on both $7^{\text {th }}$ and $28^{\text {th }}$ day.
Specimens given combination dressings of Calcium Hydroxide and honey exhibited no inflammation or very less inflammation on $7^{\text {th }}$ and $28^{\text {th }}$ day. This could be related to the anti-inflammatory, antibacterial and healing properties of honey. It has anti-inflammatory effects because it reduces hyperemia, edema and exudation by absorbing fluid from the wound. Antibacterial properties of honey are due to hygroscopic activity, acidic $\mathrm{pH}$ and hydrogen peroxide produced enzymatically. ${ }^{7}$ Honey has immune modulatory properties, i.e. it can regulate proliferation of immune cells, production of cytokines and induce the mechanism of wound repairing. ${ }^{16}$

Although a number of advanced materials are available in the market to replace the $\mathrm{CaOH}$ for its shortcomings, but they are expensive. Therefore, honey can be good choice due to its antiinflammatory, antimicrobial and wound healing properties. It shows beneficial biological properties, hence, it can lead to more favourable prognosis after pulpotomy. Normally inflammatory pulp response to any procedure or material was likely to be different to that of a compromised pulp as a result of caries, cracks, breakdown of restorations that allow bacteria to penetrate the tooth, irritate the pulp and initiate the uncontrolled inflammatory response. So a careful consideration is essential to adapt these favourable results in clinical situations. 


\section{Conclusion}

It is concluded from the findings that honey alone and $\mathrm{Ca}$-hydroxide alone almost have similar anti-inflammatory potential, but when they are used in combination, they act as more potent anti-inflammatory agents after pulptomy.

\section{Acknowledgements}

All the authors acknowledge the support extended by staff of Department of Operative Dentistry, De'Montmorency College of Dentistry Lahore, Pakistan and all the study subjects.

\section{Conflict of interest}

The author declares no conflict of interest.

\section{References}

1. Caicedo R, Abbott PV, Alongi DJ, et al. Clinical, radiographic and histological analysis of the effects of mineral trioxide aggregate used in direct pulp capping and pulpotomies of primary teeth. Aust Dent J. 2006;51(4):297-305.

2. Magnusson B. Attempts to predict prognosis of pulpotomy in primary molars. Scand J Dent Res. 1970;78(3):232-240.

3. Ranly DM. Pulpotomy therapy in primary teeth: new modalities for old rationales. Pediatr Dent. 1994;16(6):403-409.

4. Eidelman E, Holan G, Fuks AB. Mineral trioxide aggregate vs. formocresol in pulpotomized primary molars: a preliminary report. Pediatr Dent. 2001;23(1):15-18.

5. Myers DR, Shoaf HK, Dirksen TR, et al. Distribution of 14C-formaldehyde after pulpotomy with formocresol. J Am Dent Assoc. 1978;96(5):805-813.

6. Primosch RE, Glomb TA, Jerrell RG. Primary tooth pulp therapy as taught in Predoctoral pediatric dental programs in the United States. Pediatr Dent 1997;19(2):118-122.
7. Molan PC. Potential of honey in the treatment of wounds and burns. Amer J Clin Dermato. 2001;2(1):13-19.

8. Molan PC. Why honey is effective as medicine. The scientific application of its effects. Bee World. 2001;82(1):22-40.

9. Elbagoury EF, Fayed NA. Application of" natural honey" after surgical removal of impacted lower third molar. Egypt Dent J. 1985;31(3):203-211.

10. Khanal B, Baligha M, Uppal N. Effect of topical honey on limitation of radiation induced oral mucositis: an intervention study. Int J Oral Maxillofac Surg. 2010;39(12):1181-1185.

11. Khan SA, Cheema S, Qureshi RP. Evaluation of honey as a root canal disinfectant. Proceeding Sheikh Bayed Postgrad Med Inst. 2004;18(2):9195.

12. Sobhi MB, Manzoor MA. Efficacy of camphorated paramonochlorophenol to a mixture of honey and mustard oil as a root canal medicament. $J$ Coll Phys Surg Pak. 2004;14(10):585-588.

13. Elbagoury EF, Rasmy S. Antibacterial action of natural honey on anaerobic bacteroides. Egypt Dent J. 1993;39(1):381-386.

14. Giro EMA, Gondini JO, Hebling H, et al. Response of human dental pulp to calcium hydroxide paste preceded by corticosteroid/antibiotic dressing agent. Braz J Oral Sci. 2010;9(3):373-344.

15. Parolia A, Kundabala M, Rao NN, et al. A comparative histological analysis of human pulp following direct pulp capping with Propolis, mineral trioxide aggregate and Dycal. Aus Dent J. 2010;55(1):59-64.

16. Ahuja A, Ahuja V. Apitherapy-A sweet approach to dental diseases-Part I: Honey. J Adv Dent Res. 2010;1(1):81-86. 